\title{
STRESS AND SELF-EFFICACY AS SPECIFIC PREDICTORS OF SAFETY AT WORK IN THE AVIATION SECTOR
}

\author{
Magdalena Ślazyk-Sobol ${ }^{1}$, Małgorzata Dobrowolska², Joanna Zomerfeld ${ }^{2}$, Anna Pieloch² \\ ${ }^{1}$ University of Wroclaw, Wrocław, Poland \\ Institute of Psychology \\ ${ }^{2}$ Silesian University of Technology, Gliwice, Poland \\ Institute of Education and Communication Research
}

\begin{abstract}
Background: The article addresses the issue of attitudes towards safety at work in the context of subjective variables such as psychological stress and the subjective self-efficacy of workers in the aviation sector. The research was exploratory in nature. It focused on capturing the individual experiences of ground handling staff at Polish and Slovak airports. Among the 326 people surveyed were engineers, firefighters, mechanics and electricians - a crew that works in difficult and threatening working conditions on a daily basis, often experiencing unexpected accidents and breakdowns. The psychosocial factors in industry 4.0 and this branch of industry itself are - according to the authors - verified empirically quite poorly. Taking care of safety at work and strengthening a positive attitude towards safety seems to be a key aspect of management in such units. Material and Methods: The Polish Questionnaire of Attitude towards Safety by M. Znajmiecka-Sikora, the Generalized Self-Efficacy Scale and the Plopa and Makarowski Stress Feeling Questionnaire were used in the research. Results: The statistical analyses carried out, including regression analyses and a model of analysis of mediation between variables, have indicated that this sense of effectiveness is a key positive predictor of every aspect related to strengthening attitudes towards safety (a cognitive aspect $[\beta=0.21, p<0.001]$, an affective aspect $[\beta=0.15, p=0.001]$ and a behavioral aspect $[\beta=0.15, p=0.002])$, as well as the overall level of attitude towards safety $(\beta=0.19, p<0.001)$. Conclusions: The sense of effectiveness is an important mediator between the level of perceived stress and attitudes towards safety, which means that as the subjectively perceived sense of self-efficacy increases, the level of experienced stress decreases and thus the positive attitude towards safe actions and behaviors in the workplace is strengthened. Med Pr. 2021;72(5):479-87
\end{abstract}

Key words: attitudes, occupational stress, 4.0 industry, aviation sector, feeling of self-efficacy, attitudes towards safety

Corresponding author: Magdalena Ślazyk-Sobol, University of Wroclaw, Institute of Psychology, Dawida 1, 50-529 Wrocław, Poland, e-mail: magdalena.slazyk-sobol@uwr.edu.pl

Received: December 30, 2020, accepted: September 28, 2021

\section{INTRODUCTION}

Employee attitudes, crucial for the implementation of safety policies and compliance within the established principles of cooperation in industrial plants, have long been studied by social psychologists $[1,2]$.

Attitudes in general are presented in psychology literature as a special aspect of human functioning. They are defined as determining the motives for human behavior, individual choices and decision-making mechanisms. Attitudes comprise a relatively constant set of instructions that guides an individual to take a specific position (positive or negative) towards a given objective, which may be an object, as defined by social psychology, an event, an opinion and/or other people. In determining an attitude, it is important to determine its position (positive or negative), its intensity (large or small), as well as its strength, validity, compatibility and relation to other attitudes [1].

Social psychologists present a 3-factor definition for attitude, i.e., the presence of cognitive, emotional (affective) and behavioral components. The cognitive component entails the knowledge of an individual and the resulting beliefs about the posture object. The affective component contains feelings (positive or negative) about the attitude object. The behavioral component is the consequence (effect) of the cognitive and affective components $[3,4]$.

An employee attitude towards work safety can be defined as the total of relatively permanent dispositions to perceive and evaluate the principles of work safety, to react emotionally to them and to perform work safely $[3,4]$.

Funding: the publication is financed within the framework of the programme titled "Dialogue" introduced by the Minister of Science and Higher Education between 2016-2019. 
The cognitive component of attitude defined in this way refers to the knowledge of the principles and ways of performing work safely, of the dangers inherent in certain work processes and of potential accident situations. Such knowledge may be provided to the employee, among others, during instructions (initial, positional), periodic training, by means of positional manuals, risk assessment cards and other documents defining the principles of safe performance at work in the organization, and interviews with superiors and colleagues. The affective component is referred to in literature as a manifestation of an individual's emotional attitude towards compliance with health and safety rules, while the behavioral component is, in turn, expressed by undertaking given behaviors at work. For example, an employee's knowledge of the risks and ways of personal protection, coupled with a positive attitude can result in safe behavior at the workplace, in accordance with the health and safety regulations in place at the organization [2,5-7].

The issue of attitudes towards safety at work should be analyzed by taking into account the working conditions, i.e., the background of the organization, the type of machinery used, the technical condition of said machinery and equipment, the way in which the workers are organized, the presence or absence of time pressure, and the correct flow of information.

Empirical studies have shown unequivocally that as working conditions and work organization improve, the level of attitudes to safety expressed by employees increases. What is worth emphasizing is that, in contrast to the findings made by psychologists, an important parameter strengthening the observance of safety rules is also the size of the company. Large companies are associated with a higher level of the cognitive component of attitude and the presentation of safer behaviors by employees in the workplace [4,6-7]. Among the organizational factors related to attitudes towards safety and related behaviors in the workplace is an organizational climate in which safety attitudes act as a mediator: the organizational climate has a significant impact on safety and the conformance of employee attitudes to the organization's health and safety procedures.

What also determines the attitudes to safety at work are individual circumstances. Among the most important subjective characteristics associated with attitudes towards safety are age and education. The knowledge of and attitude towards safety at work are related to the employee's age: the older the employee, the greater the knowledge of and positive attitudes towards safety. In conclusion, as employees get older, they become more interested in safety at work $[3,4]$.

In most psychological research, the monitoring of employee attitudes towards safety is conducted by means of observations at the workplace, interviews or specific methods for studying the organizational climate. In the present study, the Questionnaire of Attitude towards Safety $(\mathrm{KPwB})$ by K. Znajmiecka-Sikora $[3,4]$ was used.

A subjective variable that can significantly affect the willingness of an individual to take actions that promote safety at work and strengthen one's sense of self-efficacy in this respect is perceived self-efficacy, a concept introduced by Albert Bandura [8]. Nowadays, it is described as a key resource in terms of strengthening individual self-efficacy, e.g., in the context of pro-healthy behavior, but also in the context of undertaking intentional activities $[8,9]$.

According to the social and cognitive theory, human behavior is guided by situation-outcome, action-outcome and self-efficacy expectations. Self-efficacy refers to the action itself and is part of the control of personal action $[8,10]$. A structure defined in this way is an image of individual competence.

The stronger and more effective the workers' convictions about their own effectiveness are, the higher the objectives they undertake and the stronger their commitment to the intended behavior even in the face of potential difficulties and failures. A strong sense of competence affects not only intellectual achievements, but also the efforts made and the level of determination of an individual. Significantly, work and organizational psychologists have concluded that a sense of self-efficacy affects choices in a given situation, i.e., its rejection or acceptance, depending on the expected consequences. The cognitive processes that precede action allow for the proper use of available resources and the preparation of concrete action plans. When a person experiences zero self-efficacy, his/her motivation potential automatically decreases. The first empirical research on the self-efficacy variable assumed that it allows to predict intentions and actions in different areas of human activity, including health behavior [9-13]. A higher sense of self-efficacy increases motivation to act and is associated with better individual performance.

Perceived self-efficacy not only helps to cope with stress, but also has a mobilizing effect on the functions of the immune system. According to researchers, people with a high sense of effectiveness are better able to control pain than people with low self-efficacy. The sense of 
self-efficacy is also a subjective belief that creates a variety of personal and situational elements. This may suggest that having a high sense of self-efficacy strengthens the readiness of an individual to comply with the requirements of work and the principles adopted in the organization. The strength of perceived self-efficacy expresses the degree of confidence and trust in the competences one possesses. The authors of this article have assumed that manifesting a high level of self-efficacy will strengthen one's attitudes towards safety and the observance of health and safety principles in an organization.

The concept of stress is commonly used to refer to external circumstances, requirements, burdens and difficult situations, or to describe unpleasant emotional experiences, tension and discomfort $[14,15]$. These 2 ways of understanding stress are reflected in theoretical proposals and research on the subject. Contemporary concepts of psychological stress do not only locate the sources of stress in individuals or their environment, but rather indicate a specific type of relationship (interaction, transaction) between the individual and their environment. The cognitive and transactional paradigm of stress and coping by Lazarus focuses precisely on human activity in a specific situational context. A transaction between a person and their environment constitutes, according to the author, a new quality, which is, in fact, constantly changing. It is also subject to cognitive evaluation and has a dynamic character (primary evaluation). The individual assesses those elements of their relationship with the environment that are important to him/her from the perspective of his/her own well-being.

Lazarus and Folkman [16,17] define stress as the relationship between a person and his/her environment, which is assessed by an individual as exceeding resources and threatening his/her well-being. A stress transaction in a primary assessment can be considered as an injustice, loss, threat or challenge. On each of these levels, characteristic emotions make their appearance. The perception of a situation as full of stress, known in psychology literature as "harm - loss," causes anger, regret and sadness. Threat is connected with fear and worry. Challenge is accompanied by positive emotions including hope, excitement, cheerfulness, and negative effects similar to those experienced by threat. Secondary evaluation is a cognitive process that occurs when an individual considers a relationship to be stressful. This stage of assessment involves interpreting sources of stress and estimating one's own resources. If an individual assesses their own resources as sufficient to deal with stressors, there may be a change in the primary assessment from "threat" to "challenge." The secondary assessment is, therefore, the stage of analysis that can initiate activity aimed at changing the stress transaction.

Lazarus, Folkman and Moskowitz describe coping with stress as the changing cognitive and behavioral efforts of an individual to master specific external and internal requirements [16-18]. These requirements are burdensome for the individual and exceed the resources available. The first coping strategy is problem orientation (instrumental, task-oriented). It consists in improving the relationship between the requirements of the environment and the capabilities of the person. The second strategy involves the self-regulation of emotions and consists in reducing unpleasant tension and relieving negative emotions. Experiencing stress, that is feeling intensified emotional tension, can generate various reactions in an individual, especially when it comes to their functioning in a professional environment. The authors assume that the experience of emotional tension, external stress (generated by the social and professional environment) and intrapsychic stress, defined by Plopa and Makarowski (the authors of the Stress Feeling Questionnaire) [19] as a confrontation of an individual with themselves, may cause cognitive, emotional and somatic effects related to the effectiveness of human functioning. In this context, the aim of the present study was to check the relationship between the level of perceived stress and the readiness of employees to demonstrate positive attitudes towards safety at work.

\section{Present study}

The subject of this research was the verification of the relationship between such variables as: the level of perceived stress defined as intrapsychic stress, emotional tension, external stress and a general sense of efficacy, and attitudes towards safety presented by employees in the aviation sector, particularly at Polish and Slovakian airports. The aim was to answer the following research questions and to verify the hypotheses resulting from the analysis of earlier studies in the psychology literature.

What is the relationship between the level of stress experienced by workers, the level of perceived self-efficacy and attitudes towards safety?

Which of the examined variables (the level of psychological stress and the sense of efficacy), and to what extent, predicts attitudes towards safety at work?

H1: A sense of efficacy will be a key variable when it comes to strengthening habits and behaviors that foster 
a constructive attitude towards safety at work, i.e., taking actions and adopting behaviors that comply with safety rules and regulations.

$\mathrm{H} 2$ : A sense of efficacy can mediate the relationship between stress and attitudes towards safety, i.e., a higher level of self-efficacy can effectively reduce stress and enhance safety behavior at work.

\section{MATERIAL AND METHODS}

\section{Sample}

The study included 326 persons employed in the aviation sector (in positions such as: safety specialist, aircraft mechanic, assistant mechanic, aircraft engineer, manager, manual worker, aviation structural mechanic, airport firefighter, chemical technician and others). Detailed information about the occupational category of each participant was not collected in the present study. All missing data were excluded from the analysis.

The average age of the participants was 39.3 years $(\mathrm{SD}=10.7)$. They had an average total work experience of 16.7 years $(\mathrm{SD}=11.3)$ and the average experience in their respective positions was 9.01 years $(\mathrm{SD}=2.5)$. The sample consisted mostly of men $(\mathrm{N}=278$; 85.3\%) working at the following airports: Pyrzowice and Szymany (Poland), and Košice (Slovakia). The participants employed at the 2 Polish airports $(\mathrm{N}=250$; $76.68 \%$ ) were predominant.

All measures were administered in the Polish and Slovak languages. All subjects were notified about the goal of the research and they agreed to participate. While conducting the study, the authors followed the recommendations of the Declaration of Helsinki.

The present research was treated as a pilot study. The authors of the article are in the process of collecting further quantitative data in order to develop a full psychometric adaptation of the tools used in the Slovak version of the study. After that, the given statistical comparison of scores and psychometric properties between the Polish and Slovak versions of the questionnaires will be fulfilled.

\section{The Questionnaire of Attitude towards Safety}

The Polish Questionnaire of Attitude towards Safety by M. Znajmiecka-Sikora is a tool for diagnosing employee attitudes towards work safety. According to the adopted theoretical concept, attitudes towards safety are understood as relatively permanent instructions to perceive and evaluate the principles of work safety, to react emotionally to them and to perform work safely $[3,4]$. The tool consists of 54 test items and a response scale in the following format: ZT (definitely yes), $\mathrm{T}$ (yes), TP (hard to say), $\mathrm{N}$ (no), and ZN (definitely not). The analyses conducted with the participation of 1300 people have indicated that the reliability of the entire tool is satisfactory (Cronbach's $\alpha$ : 0.849). Theoretical validity is also satisfactory. In particular, the factor analysis indicated the uniformity of scales, which together explain $45.72 \%$ of the results variance. The conducted statistical analyses prompted the conclusion that the tool in question displays sufficiently satisfactory psychometric properties.

An employee attitude towards work safety can be defined as a general, relatively permanent disposition to perceive and evaluate the principles of work safety, to react emotionally to them and to perform work safely. The cognitive component refers to the knowledge of the principles and ways of performing work safely, the dangers inherent in the work processes, and potential accident situations.

Sample statements from the questionnaire include the following: "When I notice that someone does not comply with general health and safety rules (e.g., in public places, at work, in school), I immediately intervene - I draw the person's attention, and if this does not work, I take appropriate steps (e.g., report to security, supervisor, etc.)," "If I do not have sufficient knowledge, experience or competence, I give up tasks that may be dangerous and risky," "I can assess and distinguish when someone is behaving in a risky manner and may endanger their own life or health and that of others."

\section{The Generalized Self-Efficacy Scale (GSES)}

The Polish adaptation of GSES (R. Schwarzer, M. Jerusalem, Z. Juczyński) refers to Bandura's [8] concepts of expectations and perceived self-efficacy. The scale consists of 10 statements. It was prepared in German in 1992 and translated into English the following year. Each question has 4 possible answers, ranging from "no" - $1 \mathrm{pt}$, to "yes" - 4 pts. The sum of all marks gives an overall indicator of self-efficacy. The outcome is that the higher the grade, the greater the self-efficacy. The scale constructed by Schwarzer and Jerusalem, presented in literature [13] has become a popular measurement tool. This is due to the need for methods to determine the described construction, which expresses important dispositional properties for predicting the behavior of an individual. On the basis of research led by Juczyński, it can be concluded that the Polish version of GES does not 
differ from the original version and has good psychometric properties. Higher self-efficacy increases motivation to act and is associated with better performance of an individual. The stronger the self-efficacy beliefs are, the higher the goals set by people and the stronger their involvement in the intended behavior, even in the face of accumulated failures.

Examples of the GSES statements include: "I can always solve difficult problems if I try hard enough," "Even when someone opposes me, I can find a way to achieve what I want," and "I can easily stick to my goals and achieve them."

The psychometric data concerning GSES are primarily based on the results of 3 German studies. The population of the first study included over 2000 young people and adults from Berlin and Dusseldorf, while the second study population was composed of 269 teachers from 10 cities. The third study covered over 3000 students. The internal reliability evaluated with Cronbach's a ranged $0.82-0.93$, while the reliability determined with the test-retest method in a group of 991 emigrants from the former German Democratic Republic amounted to 0.47 for males and to 0.63 for females. The scale was translated according to the rules developed for intercultural studies (the WHOQOL Translation Methodology) based on the English version. The positive formulation of the test statements facilitated obtaining a compliant translation.

The first studies were carried out on several dozen people aged 20-40 [20]. The core study covered a random population of 496 people aged 3-50 (mean age $=41.2$ ) The study participants originated from cities, towns and rural areas. All levels of education were represented. The correlation coefficients of each statement with the general result are high and range $0.47-0.63$, and Cronbach's $\alpha$ coefficient amounts to 0.85 . The scale reliability evaluated in a group of 85 people with a test-rest method (after 5 weeks) was 0.78 . The validity of the scale was evaluated by comparing the results for the group of 496 people with the results obtained with other methods [20].

\section{The Plopa and Makarowski \\ Stress Feeling Questionnaire}

The tool used to measure perceived stress was the Plopa and Makarowski Stress Feeling Questionnaire [19]. The tool consists of 27 items rated on a 5-point Likert scale. The individual questions included in the questionnaire form the following scales: emotional tension, alluding to experiencing high levels of anxiety, uncertainty, strong fatigue and exhaustion of resources.
The next scale measures the level of external stress, defined as experiencing stress in situations that exceed the capabilities of the individual (e.g., inadequate tasks, work area), but also the feeling of being unfairly assessed by others. It is characteristic of external stressors to induce the feelings of helplessness and loneliness. The third studied dimension is the level of intrapsychological stress, which is expressed by the lack of ability to cope with the emotional states experienced. This scale expresses pessimism, and a negative perception of oneself and the outside world.

Examples of statements used in the Stress Feeling Questionnaire are: "I feel anxiety that more and more things annoy me," "I think I am being fairly judged," "I have my plans, but I am afraid I will not implement them, because my psyche is too weak."

The reliability of the questionnaire is satisfactory. The internal compliance coefficients for the 3 scales (dimensions) are in the range of $0.70-0.81$.

\section{Data analysis}

First, the authors calculated some descriptive statistics (e.g., means, standard deviations) to summarize the results. Also, they calculated Cronbach's a to examine the reliability of the questionnaires. Cronbach's a is a well-known measure of reliability. It quantifies the consistency of a particular test, e.g., a questionnaire, or its subscales. It tells us whether the results can be reproduced using the same conditions. Alpha values of 0.60-0.70 indicate borderline internal consistency, while values $>0.70$ indicate satisfactory reliability.

Second, the authors examined relationships between variables using Pearson's $r$ correlation coefficients. The correlation analysis was extended by using linear regression. In 4 regression models, they examined whether stress and self-efficacy could predict attitudes towards safety at work.

Third, the authors examined the mediations between variables, calculating direct and indirect relationships between stress and attitudes towards safety, considering self-efficacy as a mediator variable. In general, mediation analyses help to widen the understanding of a relationship (direct effect) by investigating the underlying manner in which one variable impacts another through a mediator variable. In order to calculate direct and indirect effects, they used structural equation modeling (SEM). They also used the maximum likelihood method to estimate the regression coefficients.

All the analyses were conducted using the JASP software (JASP Team, 2020). 


\section{RESULTS}

Table 1 presents descriptive statistics for the variables under consideration, together with correlations between the variables.

The analysis showed that the cognitive component of the attitude towards safety was positively associated with emotional stress $(\mathrm{r}=0.11, \mathrm{p}=0.016)$ and with self-efficacy $(r=0.22, p<0.001)$. The affective component of $\mathrm{KPwB}$ - attitudes towards safety - was negatively correlated with general stress $(\mathrm{r}=-0.15, \mathrm{p}=0.001)$, emotional stress $(r=-0.14, p=0.002)$, external stress $(\mathrm{r}=-0.12, \mathrm{p}=0.010)$ and intrapsychic stress $(\mathrm{r}=-0.11$, $\mathrm{p}=0.017)$, and positively correlated with self-efficacy $(r=0.18, p<0.001)$. Similarly, the behavioral component of $\mathrm{KPwB}$ was negatively associated with general stress $(r=-0.10, p=0.028)$ and emotional stress $(\mathrm{r}=-0.12, \mathrm{p}=0.009)$, and positively correlated with self-efficacy $(r=0.16, p<0.001)$.

In turn, the general level of attitude towards safety was negatively correlated with general stress $(\mathrm{r}=-0.12$, $\mathrm{p}=0.012)$ and emotional stress $(\mathrm{r}=-0.14, \mathrm{p}=0.003)$, and positively correlated with self-efficacy $(r=0.21$, $\mathrm{p}<0.001$ ).

Overall, the relationship of attitude towards safety and its components with stress was weaker $(<0.20)$ than with self-efficacy $(>0.20)$.

Further analyses examined whether the overall level of stress and self-efficacy can predict attitudes towards safety at work and its sub-components. To this end, 4 linear regression models were constructed, in which subsequent variables predicted the general KPwB and its 3 components - cognitive, affective and behavioral. The regression analysis did not take the stress components as predictors due to the strong correlations between them (Table 1). The results of the regression analysis are presented in Table 2.

The regression analysis showed that self-efficacy was a positive predictor of $\mathrm{KPwB}$ components, i.e., cognitive $(\beta=0.21, \mathrm{p}<0.001)$, affective $(\beta=0.15, \mathrm{p}=0.001)$ and behavioral $(\beta=0.15, p=0.002)$, and the overall level of attitude towards safety $(\beta=0.19, \mathrm{p}<0.001)$.

At the same time, the general level of perceived stress of the surveyed workers did not allow for direct prediction of 2 out of 3 components of KPwB - cognitive $(\beta=-0.03, p=0.514)$ and behavioral $(\beta=-0.07$, $\mathrm{p}=0.138)$. This variable was also not a statistically significant predictor of the overall $\mathrm{KPwB}(\beta=-0.08$, $\mathrm{p}=0.112$ ).

The overall level of perceived stress, however, allowed to predict the third component of $\mathrm{KPwB}$, i.e., affective attitude, as a negative predictor $(\beta=-0.11, \mathrm{p}=0.014)$.

To sum up the results, it is concluded that self-efficacy and, in the case of the affective component of $\mathrm{KPwB}$, also the overall level of stress, made it possible to predict an overall $3-5 \%$ variance in terms of the overall attitude towards safety and its sub-components.

In addition, the analysis of mediation also indicated that the self-efficacy of workers can mediate between the overall level of stress experienced and attitudes towards safety and its components (Table 3). For both

Table 1. Stress, self-efficacy and attitudes towards safety - descriptive statistics and Pearson's $r$ linear correlation coefficients in the study conducted in 2019-2020 involving 326 persons employed in the aviation sector working at the following airports: Pyrzowice, Szymany (Poland) and Kosice (Slovakia)

\begin{tabular}{|c|c|c|c|c|c|c|c|c|c|c|c|}
\hline \multirow{2}{*}{ Variable } & \multirow{2}{*}{ a } & \multirow{2}{*}{ M } & \multirow{2}{*}{ SD } & \multicolumn{8}{|c|}{ Pearson's correlation } \\
\hline & & & & 1 & 2 & 3 & 4 & 5 & 6 & 7 & 8 \\
\hline 1. Overall stress level & 0.85 & 53.80 & 8.95 & - & & & & & & & \\
\hline 2. Emotional stress & 0.76 & 17.60 & 3.44 & $0.86^{* * *}$ & - & & & & & & \\
\hline 3. External stress & 0.70 & 18.30 & 3.35 & $0.83^{\star * *}$ & $0.58^{* * *}$ & - & & & & & \\
\hline 6. Cognitive component of $\mathrm{KPwB}$ & 0.77 & 16.55 & 3.67 & -0.08 & $-0.11^{*}$ & -0.06 & -0.02 & $0.22^{* * *}$ & - & & \\
\hline 7. Affective component of KPwB & 0.78 & 18.16 & 4.54 & $-0.15^{\star *}$ & $-0.14^{* *}$ & $-0.12^{*}$ & $-0.11^{*}$ & $0.18^{* * *}$ & $0.65^{* * *}$ & - & \\
\hline 8. Behavioral component of $\mathrm{KPwB}$ & 0.61 & 17.21 & 2.78 & $-0.10^{*}$ & $-0.12^{\star *}$ & -0.08 & -0.05 & $0.16^{\star * *}$ & $0.76^{\star * *}$ & $0.68^{* * *}$ & - \\
\hline 9. General KPwB & 0.80 & 51.92 & 8.38 & $-0.12^{*}$ & $-0.14^{\star *}$ & -0.09 & -0.06 & $0.21^{\star * *}$ & $0.92^{\star * *}$ & $0.84^{* * *}$ & $0.91^{* * *}$ \\
\hline
\end{tabular}

$\mathrm{KPwB}$ - the Questionnaire of Attitude towards Safety.

$\alpha$ - Cronbach's $\alpha$ internal compliance factor.

${ }^{*} \mathrm{p}<0.05 ;{ }^{* *} \mathrm{p}<0.01 ;{ }^{* * *} \mathrm{p}<0.001$. 
Table 2. Stress and self-efficacy as predictors of attitude towards safety and its components - results of linear regression analysis in the study conducted in 2019-2020 involving 326 persons employed in the aviation sector working at the following airports: Pyrzowice, Szymany (Poland) and Kosice (Slovakia)

\begin{tabular}{|c|c|c|c|c|c|c|}
\hline \multirow{2}{*}{ Predictor } & \multicolumn{4}{|c|}{ Linear regression analysis of the researched models } & \multicolumn{2}{|c|}{ Model assessment } \\
\hline & $\beta$ & SE & $\mathrm{t}$ & $\mathrm{p}$ & $\mathrm{R}^{2}$ & $F(d f 1, d f 2)$ \\
\hline \multicolumn{7}{|l|}{ Model 1} \\
\hline stress & -0.03 & 0.05 & -0.65 & 0.514 & & \\
\hline self-efficacy & 0.21 & 0.05 & -4.58 & $<0.001$ & & \\
\hline intercept & & & 10.48 & $<0.001$ & 0.04 & $10.67(2,469)^{* * *}$ \\
\hline stress & -0.11 & 0.02 & -2.46 & 0.014 & & \\
\hline self-efficacy & 0.15 & 0.04 & -3.28 & 0.001 & & \\
\hline \multicolumn{7}{|l|}{ Model 3} \\
\hline self-efficacy & 0.15 & 0.04 & 3.15 & 0.002 & & \\
\hline \multicolumn{7}{|l|}{ Model 4} \\
\hline intercept & & & -8.85 & $<0.001$ & 0.05 & $12.24(2,469)^{\star * *}$ \\
\hline stress & -0.08 & 0.05 & -1.59 & 0.112 & & \\
\hline self-efficacy & 0.19 & 0.12 & -4.23 & $<0.001$ & & \\
\hline
\end{tabular}

The standardized values for regression directional coefficients $(\beta)$, standard error of regression coefficient (SE), Student's t test for significance ( $t$ ), probability values (p), coefficient of determination $\left(\mathrm{R}^{2}\right)$, Snedecore's $F$ with overall significance of the regression model (F) and its degrees of freedom (df).

Models: 1 - Explained variable: cognitive component of the Questionnaire of Attitudes towards Safety (KPwB); 2 - Explained variable: affective component KPwB;

3 - Explained variable: behavioral component $\mathrm{KPwB} ; 4$ - Explained variable: general KPwB.

$* * * \mathrm{p}<0.001$.

Table 3. Self-efficacy as a mediator of relations between stress and attitudes towards safety - results of a mediation analysis in the study conducted in 2019-2020 involving 326 persons employed in the aviation sector working at the following airports: Pyrzowice, Szymany (Poland) and Kosice (Slovakia)

\begin{tabular}{|c|c|c|c|c|c|c|c|c|c|}
\hline \multirow{2}{*}{ KPwB } & \multicolumn{9}{|c|}{ Effect } \\
\hline & b $(95 \% \mathrm{CI})$ & $\mathrm{Z}$ & $\mathrm{p}$ & b $(95 \% \mathrm{CI})$ & Z & $\mathrm{p}$ & b (95\% CI) & Z & $\mathrm{p}$ \\
\hline Cognitive component & $-0.01(-0.06-0.03)$ & -0.66 & 0.512 & $-0.02(-0.03-(-0.01))$ & -3.33 & $<0.001$ & $-0.04(-0.08-0.01)$ & -1.66 & 0.097 \\
\hline Affective component & $-0.04(-0.07-(-0.01))$ & -2.47 & 0.014 & $-0,01(-0.02-(-0.00))$ & -2.72 & 0.014 & $-0.05(-0.08-(-0.02))$ & -3.22 & 0.001 \\
\hline
\end{tabular}

Abbreviation as in Table 1.

$\mathrm{b}$ - unstandardized regression coefficient.

The maximum likelihood method was used to estimate the parameters of the model.

$\mathrm{KPwB}$ and its 3 sub-components, indirect effects were statistically significant: for the cognitive component: $\mathrm{b}=-0.02$ (95\% CI: $-0.03 ;-0.01), \mathrm{p}<0.001$; for the affective component: $\mathrm{b}=-0.01$ (95\% CI: $-0.02 ; 0.00)$, $\mathrm{p}=0.014$; for the behavioral component: $\mathrm{b}=-0.01$ (95\% CI: $-0.02 ; 0.00), \mathrm{p}=0.008$; and for the overall KPwB: $\mathrm{b}=-0.04$ (95\% CI: $-0.07 ;-0.02), \mathrm{p}=0.001$.
It was observed, therefore, that as the general sense of stress of the subjects decreased, their results in terms of self-efficacy ( $\beta=-0.09, \mathrm{p}<0.001)$ increased, which was associated with an increase in their results in terms of the cognitive $(\beta=0.25, p<0.001)$, affective $(\beta=0.12$, $\mathrm{p}<0.001)$ and behavioral components $(\beta=0.13, \mathrm{p}=0.002)$, as well as general $\mathrm{KPwB}(\beta=0.51, \mathrm{p}<0.001)$. 


\section{DISCUSSION}

The results of the empirical research carried out in a group of airport ground handling staff present interesting material which, according to the authors, should be applied in practice in activities directly supporting the examined professional group (industry).

Statistical analyses have confirmed the negative relationship between stress levels and general attitudes towards safety and its components (cognitive, affective and behavioral). Attention should be paid, however, to the stronger positive relationship between the sense of self-efficacy and general attitudes towards safety. A higher level of self-efficacy is directly proportionally related to the higher results of those tested in safety attitudes scales.

As far as the analyses of regression models are concerned, in this research the strongest and most important predictor of attitudes towards safety at work is the sense of self-efficacy. The level of stress experienced by workers only makes it possible to anticipate the affective component of $\mathrm{KPwB}$ in a negative way as well, which means that a higher level of stress will weaken the workers' resolve to respect safety regulations at work. The results of the study remain consistent with other reports on the relationship between safety attitudes and individual circumstances.

The attitude towards safety according to ZnajmieckaSikora $[4,5]$ is related to the tendency to take both instrumental and stimulus risks. The direction of this relationship is negative, which means that an increase in the attitude towards safety and all its 3 components is accompanied by a lower propensity to take risks. Conversely, the higher the propensity to take both stimulus and instrumental risks, the more negative the attitude towards safety.

It is worth mentioning that the sense of where control is located, i.e., the conviction that the effects of one's own work relate to internal or external factors, is also linked to an attitude towards safety. The direction of these correlations is negative, which means that the higher the external sense of where control is located, the lower the results in terms of attitudes towards safety. In turn, a more internally located sense of control (the locus of control) fosters more positive attitudes towards work. In light of data from empirical studies, subject variables such as the sense of self-efficacy or this internal sense of control become important predictors of attitudes towards safety at work.

The authors' own research confirmed the first hypothesis concerning the mediation character of the variable: the sense of self-efficacy. This is an important mediator of the relationship between the level of perceived external stress, intrapsychic stress and emotional tension, and safe behavior in the workplace. What seems to be a very important conclusion in practical terms is that the perceived level of stress decreases with the increase in the sense of self-efficacy, which at the same time stimulates the level of attitude related to observing safety regulations at work. Literature on the subject has already proven a positive relationship between the sense of self-efficacy and the strengthening of pro-health behaviors, which seems to be very important in the case of numerous preventive and rehabilitation measures $[8-10,13,14]$.

As far as promoting the principles of safety at work is concerned, strengthening the readiness of employees to comply with the established rules and regulations and driving home the importance of safety for the overall functioning of the organization, but also for the health and well-being of the staff, the sense of self-efficacy expressed by employees is a key subject variable supporting the creation of a safety culture. It would be worthwhile to strengthen the perceived self-efficacy among employees in the aviation sector through individualized development programs, but also through a well-thought-out and balanced personnel policy (e.g., feedback processes, employee assessments, motivation programs). Higher indicators associated with the conviction of one's ability to achieve objectives and work effectively translate to more positive attitudes among employees towards safety, especially in a sector as dependent on safety as aviation.

\section{CONCLUSIONS}

The study presented in this paper was conducted prior to the COVID-19 pandemic, which makes it impossible to generalize the conclusions in the studied sector to bigger groups of employees in the same sector. A significant methodological limitation of the study was the use of questionnaires. These methods provide limited data. It was impossible, however, to find a more effective method enabling us to reach such a large group of employees in such a "closed" sector.

The authors' scientific interests are connected with attitudes, behaviors and relations between employees and their professional environment in the wider context of work 4.0, especially in the sectors of cutting-edge technologies, automation and industry 4.0.

The Fourth Digital (Industrial) Revolution, called industry 4.0, brings a number of changes. The term applies to the industry only metaphorically. It is a philosophy 
of business organization and management using new technologies. The observed technological and social progress is naturally reflected in the organization functioning, new 4.0 work environments and new staff organic and inorganic teams.

Further studies will focus on the adaptation of scientific instruments to the specifics of this sector, especially through concentrating on the nature of working in cutting-edge technology industries, and the challenges associated with them.

\section{REFERENCES}

1. Wojciszke B. Social Psychology. Warszawa: Scholar Publishing House; 2013.

2. Nowak S. The concept of attitude in theories and applied social research. In: Nowak S, editor. Theories of attitudes. Warszawa: Scientific Publisher PWN; 1973. p. 20-29.

3. Znajmiecka-Sikora M. Assessment of the safety culture in the MIŚP sector based on surveys of enterprises from the Łódź region. In: Juźwicka A, Szymańska K, Walecka A, editors. A new look at organizational culture. Łódź: Publishing House of the Lodz University of Technology; 2014. p. 53-62.

4. Znajmiecka-Sikora M, Boczkowska K. Analysis of safety culture on the example of selected Polish production enterprises. In: Arezes PM, Baptista JS, Barroso MP, Carneiro P, Cordeiro P, Costa N, et al., editors. Occupational Safety and Hygiene IV. Londyn: CRC Press; 2016. p. 349-352.

5. Szczygielska A. Factors of the work environment and the cognitive component of employees' attitude towards work safety. In: Doliński D, Maciuszek J, Polczyk R, editors. Around social impact. Krakow: UJ Publishing House; 2012. p. 180-198.

6. Stasiła-Sieradzka M. The participation of work and organizational psychology in creating a safe work environment. In: Lewandowski J, Znajmiecka-Sikora M, editors. Modern standards in the field of occupational health and safety management. Opportunities and threats. Łódź: Publishing House of the Lodz University of Technology; 2012. p. $138-160$.
7. Stasiła-Sieradzka M, Dobrowolska M. Study of the work safety climate as an element of benchmarking in safety management in the organization. Society and education. 2016;21(2):471-487.

8. Bandura A. Self-Efficacy The Exercise of Control, New York: Freeman; 1997.

9. Schwarzer R. Psychologie des Gesundheitsverhaltens. Göttingen: Hogrefe; 1992.

10. Schwarzer R. Measurement of Perceived Self-efficacy: Psychometric Scales for Cross-cultural Research. Berlin: Freie Universität Berlin, Institut für Psychologie; 1993.

11. Schwarzer R. Self-efficacy in undertaking and continuing health behaviors. Previous theoretical approaches and a new model. In: Heszen-Niejodek I, Sęk H, editors. Psychology of health. Warszawa: PWN; 1997. p. 175-205.

12. Schwarzer R. General Perceived Self-Efficacy in 14 Cultures, An Electronic Volume. Produced for the European Health Psychology Society, 1998.

13. Schwarzer R, Fuchs R. Self-efficacy and Health Behaviours. In: Conner M, Norman P, editors. Predicting Health Behaviour. Buckingham - Philadelphia: Open University Press; 1996. p. 163-196.

14. Heszen-Niejodek I. Style of coping with stress: facts and controversy. Psychol J. 1997;1:7-22.

15. Heszen I, Sęk H. Health and stress. In: Strelau J, Doliński D, editors. Academic psychology. Coursebook. Volume 2. Gdańsk: Psychological Publisher; 2010. p. 682-734.

16. Lazarus RS, Folkman S. Stress, appraisal and coping. New York: Springer - Verlag; 1984.

17. Lazarus RS. From psychological stress to emotions: A history of changing outlooks. Annu Rev Psychol. 1993;44:1-21. https://doi.org/10.1146/annurev.ps.44.020193.000245.

18. Folkman S, Moskowitz J. Positive affect and the other side of coping. Am Psychol. 2000;55:647-654.

19. Plopa M, Makarowski M. Feeling of Stress Questionnaire. Warszawa: Vizja Press\&IT; 2010.

20. Juczyński Z. Psychological determinants of health behaviors on the example of adult research. In: Łazowski J, Dolińska-Zygmunt G, editors. Towards better functioning in health and disease. Wrocław: AWF Publishing House; 1997. p. 285-291.

This work is available in Open Access model and licensed under a Creative Commons Attribution-NonCommercial 3.0 Poland License - http://creativecommons.org/licenses/by-nc/3.0/pl/deed.en. 